
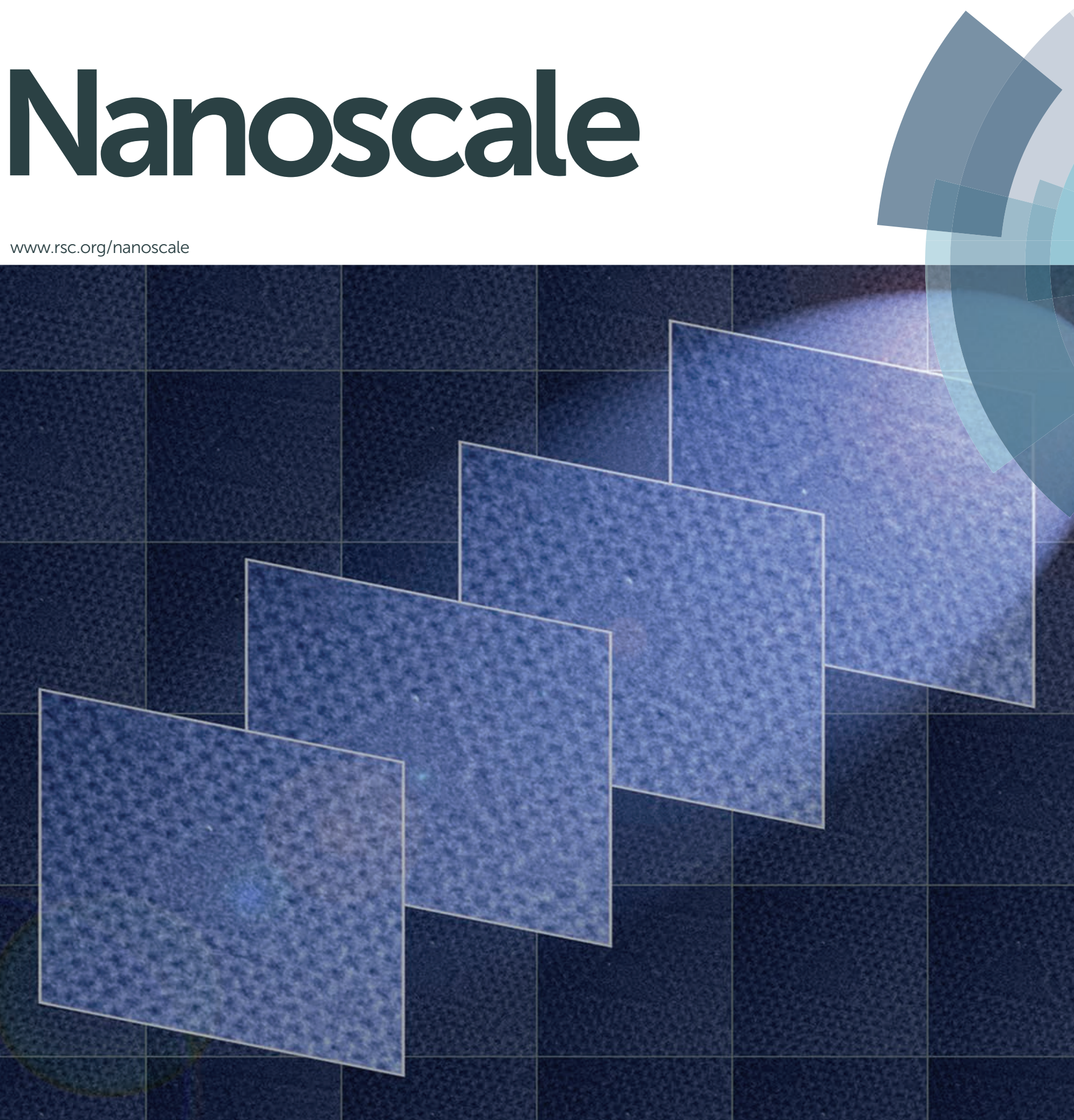

ISSN 2040-3364

Atomic-scale dynamics of triangular hole growth in monolayer hexagonal boron nitride under electron irradiation 


\title{
Nanoscale
}

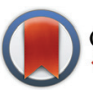

CrossMark \& click for updates

Cite this: Nanoscale, 2015, 7, 10600

\section{Atomic-scale dynamics of triangular hole growth in monolayer hexagonal boron nitride under electron irradiation $\uparrow$}

\author{
Gyeong Hee Ryu,,$^{\mathrm{a}}$ Hyo Ju Park,,$^{\mathrm{a}}$ Junga Ryou, ${ }^{\mathrm{b}}$ Jinwoo Park, $^{\mathrm{b}}$ Jongyeong Lee, ${ }^{\mathrm{a}}$ \\ Gwangwoo Kim, ${ }^{d}$ Hyeon Suk Shin, ${ }^{\text {d,e,c,f }}$ Christopher W. Bielawski, ${ }^{\text {c,d,e }}$ \\ Rodney S. Ruoff, ${ }^{c, d, a}$ Suklyun Hong ${ }^{\star b}$ and Zonghoon Lee ${ }^{\star a, c, f}$
}

\begin{abstract}
The production of holes by electron beam irradiation in hexagonal boron nitride (hBN), which has a lattice similar to that of graphene, is monitored over time using atomic resolution transmission electron microscopy. The holes appear to be initiated by the formation of a vacancy of boron and grow in a manner that retains an overall triangular shape. The hole growth process involves the formation of single chains of $\mathrm{B}$ and $\mathrm{N}$ atoms and is accompanied by the ejection of atoms and bundles of atoms along the hole edges, as well as atom migration. These observations are compared to density functional theory calculations and molecular dynamics simulations.
\end{abstract}

Received 6th March 2015, Accepted 15th April 2015

DOI: $10.1039 / \mathrm{c} 5 \mathrm{nr} 01473 \mathrm{e}$

www.rsc.org/nanoscale different types of terminated edges due to the heterogeneity of $\mathrm{hBN}$, factors that have been shown to affect the material's intrinsic electrical properties. ${ }^{11}$ The growth mechanisms of extended holes in hBN are also not well established. For example, unlike graphene, where single chains of carbon atoms and related defects are well characterized, ${ }^{12-17}$ single chains made deliberately in hBN sheets via in situ production techniques (i.e., inside a transmission electron microscope) have been observed at $650{ }^{\circ} \mathrm{C} .{ }^{18}$

In this study, the growth of triangular holes is studied in large, monolayer sheets of hBN from the nucleation of a $B$ vacancy to areas that exceed $50 \mathrm{~nm}^{2}$ using atomic resolution transmission electron microscopy (ARTEM). As part of these studies, the growth dynamics of single triangular holes as well as the processes by which they merge with other holes were explored. The experimental observations were then compared with density functional theory (DFT) calculations and molecular dynamics (MD) simulations, which provided additional insight into the mechanisms of hole growth.

\section{Results and discussion}

To elucidate the mechanism of hole growth in hBN monolayers, the dynamics of triangular holes induced by electron beam irradiation were analyzed using ARTEM. A large area monolayer of hBN (grown by chemical vapor deposition and then transferred to a TEM grid) at atomic resolution is shown in Fig. 1 (Fig. S1 $\dagger$ for more detailed images). Fig. 1c shows intensity profiles of atoms in a raw image of Fig. 1a taken by 

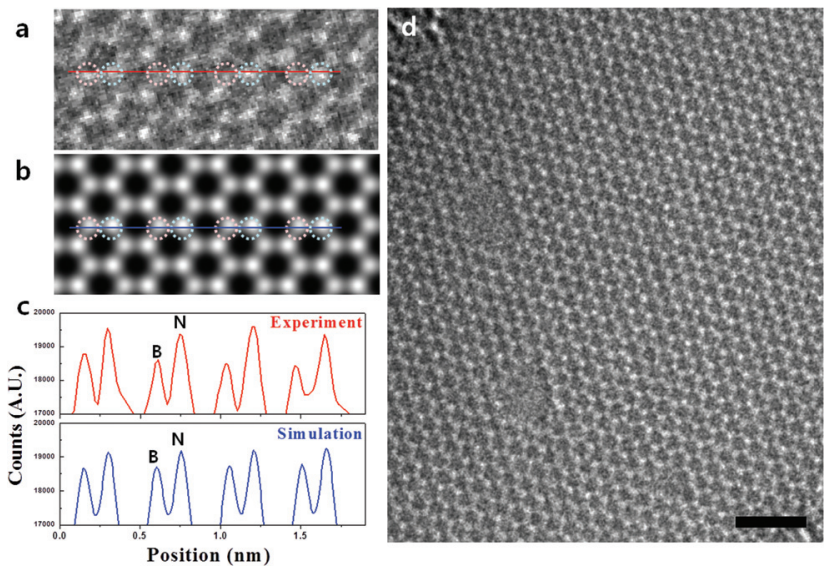

Fig. 1 (a) Raw image taken by an ARTEM and (b) simulation image at the imaging condition. (c) Intensity profiles from the trace in (a) and (b). (d) A TEM image showing a series of triangular holes in $\mathrm{hBN}$. The scale bar is $1 \mathrm{~nm}$.

an aberration-corrected TEM with a monochromator (see the Experimental section for additional details of the TEM conditions) and Fig. $1 \mathrm{~b}$ represents a simulation image under the imaging conditions. The $\mathrm{B}$ and $\mathrm{N}$ atoms can be distinguished as the $\mathrm{N}$ atoms display a $3.5 \%$ higher intensity than the $\mathrm{B}$ atoms with a $\pm 2 \%$ deviation in the real image. Because previous theoretical and experimental studies reported that triangular holes in hBN formed by electron beam irradiation have $\mathrm{N}$ terminated edges, ${ }^{11,19}$ the alternation of intensity measured from the edge atoms are in good agreement with the positions of the $\mathrm{B}$ and $\mathrm{N}$ atoms, as shown in Fig. 1d. To further evaluate the intensity of the real image, a simulation was performed under the imaging conditions using MacTempasX. Fig. 1c shows the experimental and simulation intensity profiles. The agreement indicates that the distinction of B and $\mathrm{N}$ atoms is possible from one aberration-corrected TEM image. Indeed, such optimized imaging conditions were used to acquire the results described below.

The hBN hole growth process was subsequently investigated (Fig. 2). A vacancy labeled $V_{\mathrm{B}}$, which refers to the site of a missing B atom, is shown in Fig. 2a. Through continuous electron beam irradiation, the $\mathrm{B}$ and $\mathrm{N}$ atoms were removed from sites adjacent to $V_{\mathrm{B}}$ in a manner that maintained an overall triangular shape, although some trapezoidal intermediates were observed. For example, while the removal of a pair of $\mathrm{B}$ and $\mathrm{N}$ atoms adjacent to $V_{\mathrm{B}}$ (i.e., $V_{\mathrm{B}-\mathrm{BN}}$ ) resulted in the formation of a trapezoidal site, a triangular hole re-formed upon the subsequent loss of an additional B atom (e.g., $\left.V_{\mathrm{B}-\mathrm{BN}-\mathrm{B}}\right)$, as shown in Fig. 2c. This process continued as the hole grew and involved the temporary introduction of an atom within the growing hole (cf., Fig. $2 \mathrm{~d}$ and f; see below for a deeper discussion of the migration of $\mathrm{B}$ and $\mathrm{N}$ atoms).

Efforts were then directed towards assessing whether the holes maintained their triangular shape after prolonged periods of electron beam irradiation. As summarized in Fig. 3, the growth of a triangular hole appeared to be initiated by the removal of $\mathrm{B}$ and $\mathrm{N}$ atoms near the centers of the hole edges.
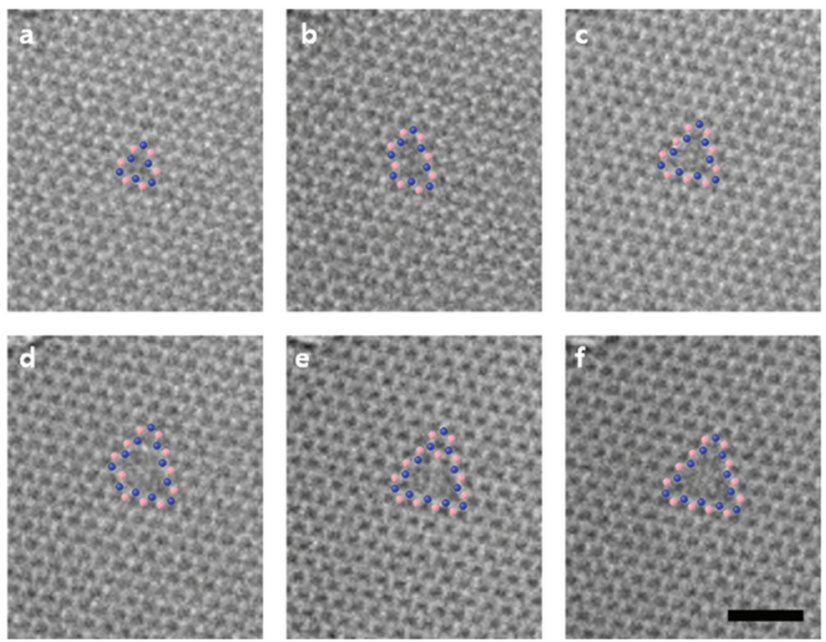

Fig. 2 The sequential hole growth process from a vacancy in monolayer hBN at atomic resolution. The process starts (a) with the development of a $B$ vacancy $\left(V_{B}\right)$ and grows as shown in (b) through ( $f$ ). Additional details can be seen in Movie S1 and Fig. S2. $\dagger$ The blue and red dots represent $\mathrm{N}$ and $\mathrm{B}$ atoms, respectively. The scale bar is $1 \mathrm{~nm}$.
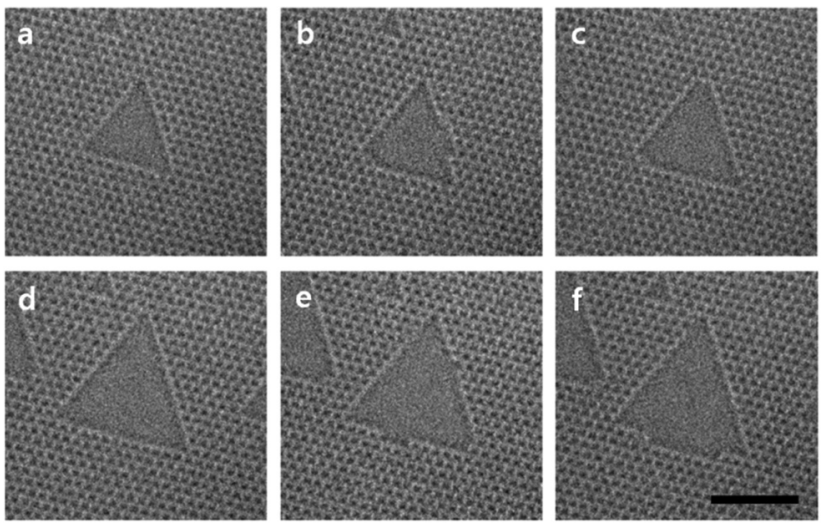

Fig. 3 Sequential atomic resolution images of monolayer hBN showing how the shape and orientation of the holes are maintained upon further growth. Additional details can be seen in Movie S2. $\dagger$ The scale bar is $2 \mathrm{~nm}$.

This experimental observation was supported by DFT calculations, which indicated that triangular holes featuring edges with paired $\mathrm{B}-\mathrm{N}$ atoms missing near the middle of an edge are more stable than those with atoms missing near a vertex (Fig. S3†). Regardless, under prolonged electron beam irradiation, the $\mathrm{B}$ and $\mathrm{N}$ atoms next to the vacancies were subsequently ejected in a manner that ultimately restored the overall triangular shape of the hole. As shown in Fig. $S 4, \dagger$ the triangular shape of the holes was maintained even after two holes merged together. In addition, exposure of an edge region to electron beam irradiation resulted in the formation of triangular holes ( $c f$., Fig. S5†). Once the electron beam irradiates within or at the edge of a hBN sheet, a $\mathrm{N}$ terminated triangular hole is unconditionally formed and its shape is maintained during the hole growth process. 
During these studies, it was noted that $\mathrm{B}$ and $\mathrm{N}$ atoms were often ejected as bundles as opposed to individual atoms, at least under the experimental conditions used. For example, as shown in Fig. S6a and S6b, $\uparrow$ bundles of B and $\mathrm{N}$ atoms were removed between successive imaging time intervals ( $\sim 0.5 \mathrm{~s})$. Because atom movement is faster than $0.5 \mathrm{~s}$, DFT calculations and MD simulations were performed to gain additional insight into this process. A summary of the calculated bond lengths between various atoms in and near a triangular hole in hBN is shown in Fig. 4a. Note that the calculated B-N bond lengths measured perpendicular to the hole edge were longer than those found along the hole edge ( $c f$., especially, positions indicated by 7 and $8 v s$. positions indicated by 5). Moreover, the difference in the calculated $\mathrm{B}-\mathrm{N}$ bond lengths was found to be consistent regardless of the hole size. Hence,

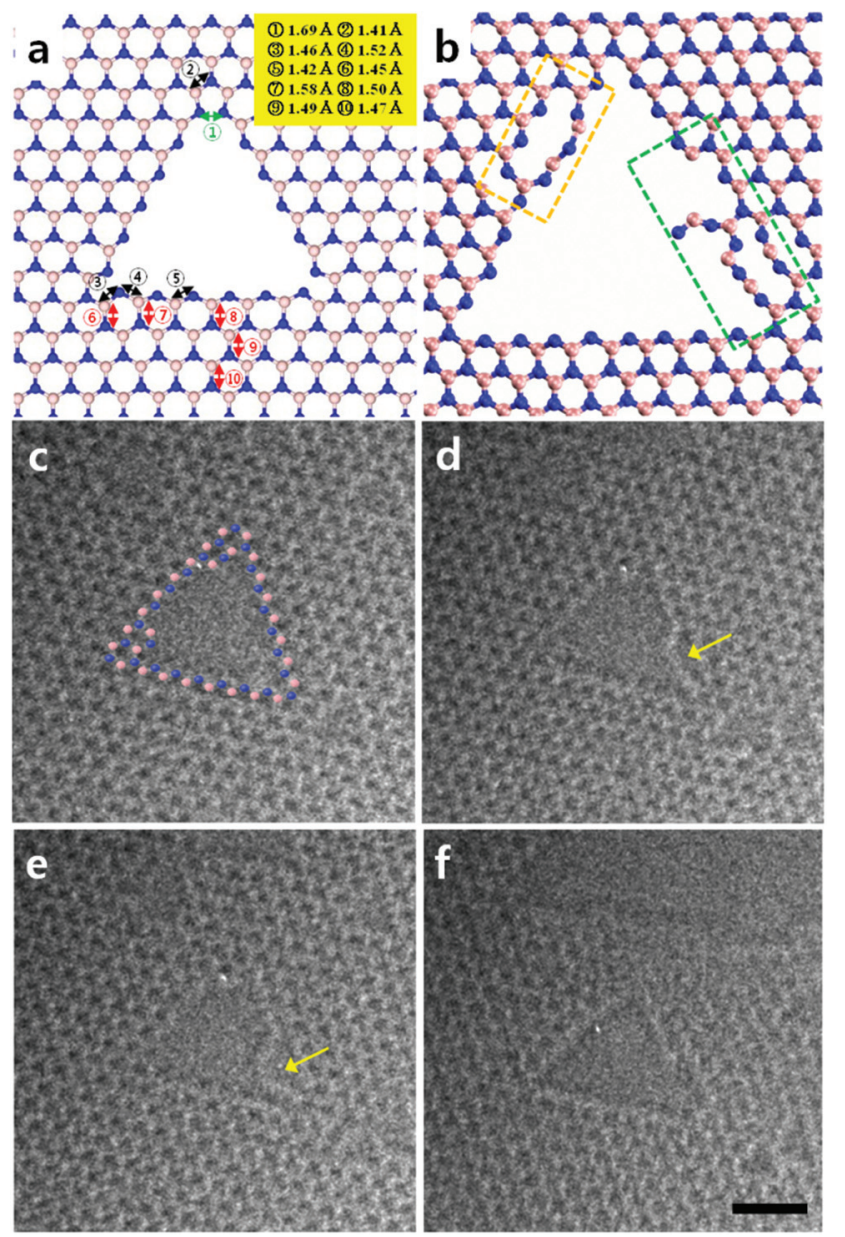

Fig. 4 (a) and (b) A summary of DFT calculations and MD simulations of hole growth processes in monolayers of $\mathrm{hBN}$. The blue and red dots represent $\mathrm{N}$ and $\mathrm{B}$ atoms, respectively: (a) Calculated bond lengths between various atoms in a triangular hole. (b) The B-N bonds perpendicular to the hole edge are broken first and often bundles of atoms are then knocked off. (c)-(f) A series of TEM images taken over time. The images show the formation of a single chain followed by its fluctuation in position and ultimate removal (see also Movie $\mathbf{S 4} \uparrow$ ). The scale bar is $1 \mathrm{~nm}$. when a $\mathrm{B}$ and/or $\mathrm{N}$ atom is ejected, the $\mathrm{B}-\mathrm{N}$ bonds perpendicular to the edge become weakened and thus are more likely to be broken upon further electron beam irradiation. The results derived from the DFT calculations were supported by MD simulations. As shown in Fig. $4 \mathrm{~b}$ and Movie S3, $\dagger$ the breaking of a B-N bond perpendicular to a hole edge generated a chain of $\mathrm{B}$ and $\mathrm{N}$ atoms, as indicated by the yellow dotted box. The chain then became detached and was subsequently knocked off as a bundle of atoms, as indicated by the green dotted box.

Direct evidence of single chain formation, as supported by DFT calculations and MD simulations, was observed by ARTEM in Fig. 4c-f. Electron beam irradiation of the triangular hole (Fig. 4c) resulted in the formation of a single chain comprised of $\mathrm{B}$ and $\mathrm{N}$ atoms (Fig. 4d). The chain detachment process appeared to originate from the breaking of a $\mathrm{B}-\mathrm{N}$ bond perpendicular to a hole edge. The chain was found to fluctuate in position (Fig. 4e) before being finally knocked off (Fig. 4f). Based on these observations, the $\mathrm{B}$ and $\mathrm{N}$ atoms do not appear to be ejected individually; rather, $\mathrm{B}-\mathrm{N}$ bonds perpendicular to a triangular hole edge may break first, resulting in the formation of a single chain of atoms that is ultimately removed. Compared to the $\mathrm{B}$ and $\mathrm{N}$ atoms found within the lattice of hBN, single chains containing the two heteroatoms are relatively unstable, and under the experimental conditions used here, they are readily removed.

To gain additional insight into the properties of single chains comprised of $\mathrm{B}$ and $\mathrm{N}$ atoms, efforts were directed toward determining whether single chains were formed during the merging of multiple holes. A series of consecutive TEM images that captured the merging of two holes are shown in Fig. 5. In general, such processes are too fast to be captured by TEM, as atoms are often ejected and/or are repositioned faster than the time elapsed between images; however, adsorbates can pin the hole and thus facilitate the capture of an intermediate state. As shown in Fig. 5d, the merging of two holes resulted in a temporal bright contrast line, which may be due to the local fluctuation of the corresponding hBN edges and/or an accelerated ejection rate. Subsequent spreading of the fluctuating region resulted in the formation of single chains of $\mathrm{B}$ and $\mathrm{N}$ atoms inside the hole (Fig. $5 \mathrm{f}$ ), followed by the development of two triangular holes (Fig. 5g). Loops and stretched chains containing a series of alternating $\mathrm{B}$ and $\mathrm{N}$ atoms were also observed (Fig. 5h-k). Collectively, these observations indicate that single chains comprised of $\mathrm{B}$ and $\mathrm{N}$ atoms may be formed when holes in hBN coalesce. Note that some of the brighter spots at the edge of the triangular holes may be due to Si atoms. Although the materials described herein were not subjected to further chemical analysis, there does not appear to be any metal residue during the synthesis or transfer processes. $^{20}$ Moreover, Si atoms are commonly observed in materials synthesized via CVD in quartz vessels. ${ }^{21}$ We conclude that the Si atoms may move around freely on the surfaces of hBN, but do not contribute to any hole growth. This conclusion was based in part on a report indicating that pure $\mathrm{Si}$ is unlikely to exhibit drilling or etching behavior in graphene. ${ }^{22}$ 

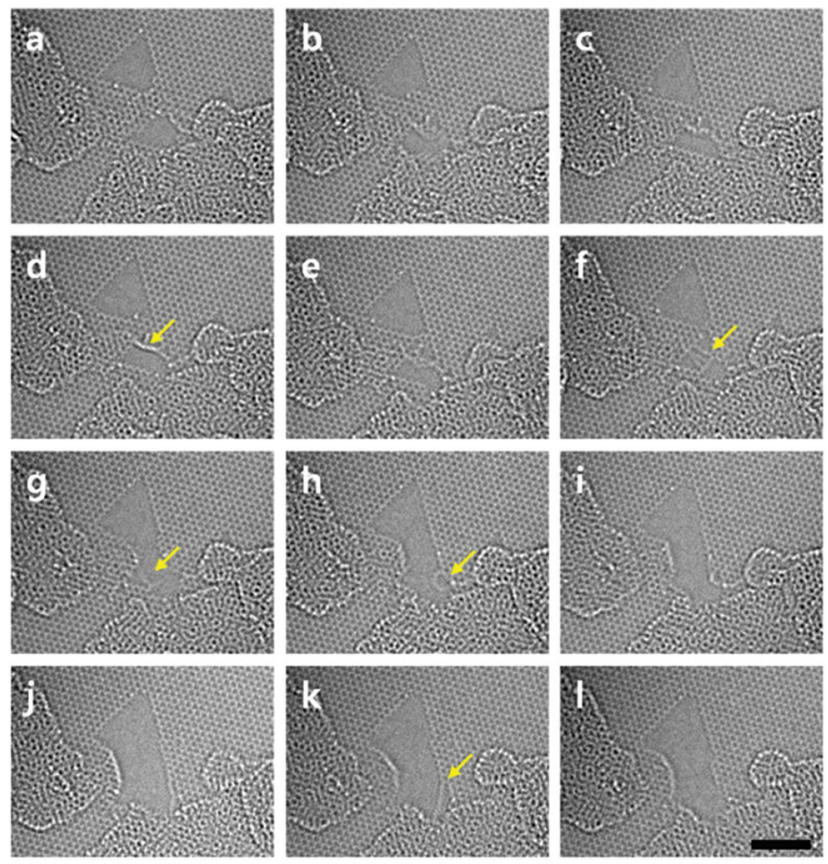

Fig. 5 A series of TEM images showing how two triangular holes merge in a monolayer of hBN. The yellow arrows indicate single chains or loops containing $\mathrm{B}$ and $\mathrm{N}$. See the text and Movie $\mathrm{S} 5 \dagger$ for additional details. The scale bar is $2 \mathrm{~nm}$.

Apart from silicon or other metal atoms, oxygen containing species may contribute to the hole formation mechanism because there is some oxygen and water vapor inside the TEM instrument even under UHV conditions. Although the chemical etching effect might be considered in understanding the damage mechanism of materials in TEM, only knock-on damage is addressed in this study. (See details of our interpretation about the contribution of oxygen containing species to the hole formation mechanism in the ESI. $\dagger$ )

As indicated in Fig. 6, the migration of atoms along the edges of holes in hBN was also evaluated over time. Although the $\mathrm{B}$ and $\mathrm{N}$ atoms may be predicted to move faster than the sampling time required for sequential imaging, a series of meta-stable configurations at the hole edges was observed. For example, the blue arrow in Fig. $6 \mathrm{~b}$ points to a series of missing $\mathrm{B}$ and $\mathrm{N}$ atom sites, whereas subsequent migrations changed

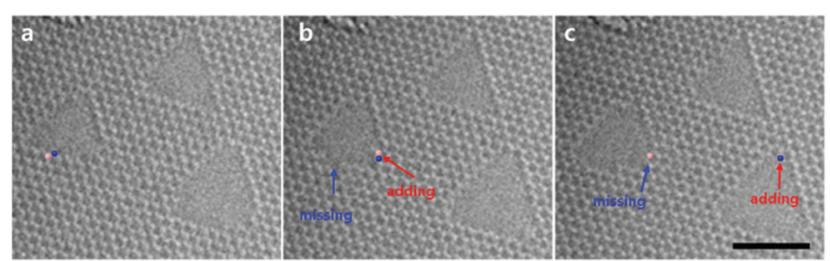

Fig. 6 A series of TEM images of triangular holes produced by the prolonged irradiation of $\mathrm{hBN}$. The blue dots represent $\mathrm{N}$ atoms, whereas the red dots represent $B$ atoms. The migration of the $B$ and $N$ atoms is observed over time. The scale bar is $2 \mathrm{~nm}$. the shape of the hole, as shown in Fig. 6c. In an earlier study, it was reported that electron beam irradiation does not induce the migration of $\mathrm{B}$ and $\mathrm{N}$ atoms in $\mathrm{hBN} ;{ }^{23}$ however, the data presented here clearly show that the migration as well as reconstruction of $\mathrm{B}$ and $\mathrm{N}$ atoms in a monolayer of $\mathrm{hBN}$ is possible. Although the addition of atoms to the hole might be derived from adventitious carbon adsorbates, it is more likely that $\mathrm{B}$ and $\mathrm{N}$ atoms migrate because the number of added atoms and missing atoms in the hBN sheet are the same and the distance between them is shorter than that between added atoms and carbon adsorbates. As such, holes may not grow but may become filled, at least partially, by the migration of B and $\mathrm{N}$ atoms over time. Collectively, these observations differ from those obtained with graphene. In graphene, various hydrocarbon adsorbates adhere to the surface and carbon impurities are often abundant so that the addition of carbon atoms to holes and/or migration is relatively more likely to occur under electron beam irradiation. While the lack of B and $\mathrm{N}$ sources intrinsically limits the addition of such atoms to holes in hBN, the migration of such atoms does appear to occur, albeit infrequently.

\section{Conclusions}

In summary, the growth of triangular holes in hBN monolayers was observed using sequential ARTEM imaging. When a monolayer of hBN was subjected to electron beam irradiation, a vacancy formed initially and grew while maintaining a triangular shape. Such shapes were observed even when such holes merged. Through a series of TEM images that were supported by DFT calculations and MD simulations, the mechanism for the growth of these holes appeared to involve the ejection of $\mathrm{B}$ and $\mathrm{N}$ atoms near the centers of the hole edges and also the ejection of bundles of atoms. Such processes involve the breaking of $\mathrm{B}-\mathrm{N}$ bonds that are perpendicular to the hole edges and result in the formation of single chains containing $\mathrm{B}$ and $\mathrm{N}$ atoms. Multiple chains containing $\mathrm{B}$ and $\mathrm{N}$ were also observed when two holes merged together, whereas previous studies have focused primarily on the observation of a single chain of $\mathrm{C}$ atoms in holes in graphene. Moreover, the migration of $\mathrm{B}$ and $\mathrm{N}$ atoms in monolayers of $\mathrm{hBN}$ was observed using ARTEM, as indicated by edge reconstruction, although such phenomena occur less frequently when compared to analogous carbon-based materials (i.e., graphene).

\section{Experimental}

\subsection{Synthesis of hBN and ARTEM observations}

The hBN specimens used in our experiments consisted primarily of monolayers and were synthesized using chemical vapor deposition. ${ }^{20}$ Conventional TEMs do not have adequate resolution for imaging single atoms of hBN and are often operated at high voltages, which lead to immediate irradiation damage in 
the specimens before reliable observations can be made. Furthermore, if experiments are performed at a low voltage such as $60 \mathrm{kV}$, knock-on damage as well as ionization damage should be considered. To avoid these problems, specimens were analyzed using an aberration-corrected FEI Titan Cube TEM (FEI Titan ${ }^{3}$ G2 60-300), which was operated at $80 \mathrm{kV}$ acceleration voltage because $80 \mathrm{kV}$ is an intermediate value of the knock-on threshold values of $\mathrm{B}(74 \mathrm{kV})$ and $\mathrm{N}(84 \mathrm{kV}) .{ }^{24}$ Therefore, our experiments mainly consider the knock-on damage. The microscope provides sub-Angstrom resolution at $80 \mathrm{kV}$ and $-21 \pm 0.5 \mu \mathrm{m}$ of spherical aberration $\left(C_{\mathrm{s}}\right)$ with a monochromator and thus is capable of imaging individual atoms within the hBN lattice. Typical electron beam densities were adjusted to around $5 \times 10^{5} \mathrm{e}^{-} \mathrm{nm}^{-2}$. We analyzed the atomic images using a white atom contrast (as opposed to a black atom contrast ${ }^{7}$ ) in order to obtain actual atom positions under properly focused conditions needed for direct image interpretation.

\subsection{Time lapse microscopy}

After acquiring a series of images using the Gatan Digital Micrograph (DM) script, structural changes in the observed area were analyzed frame-by-frame. Each of these images was taken with an exposure time of $0.5 \mathrm{~s}$ and an interval time of $1.7 \mathrm{~s}$. To facilitate the identification of the holes generated by electron beam irradiation, clean areas that were free of adsorbates and other impurities were examined.

\subsection{DFT calculations}

Density functional theory calculations were performed using the Vienna $a b$ initio simulation package (VASP). ${ }^{25,26}$ A single $k$-point ( $\Gamma$ point) for a $15 \times 15$ supercell with a cutoff kinetic energy of $400 \mathrm{eV}$ was used. The ions were represented by projector-augmented wave (PAW) potentials, ${ }^{27,28}$ and van der Waals (vdW) interactions, ${ }^{29}$ as used in Grimme's theory, were implemented into the VASP. A generalized gradient approximation was used to describe the exchange-correlation functional. $^{30,31}$ The atomic positions of all structures were relaxed until the Hellmann-Feynman forces were lower than $0.01 \mathrm{eV} \AA^{-1}$.

\subsection{MD simulations}

To explore hole growth in layers of hBN, molecular dynamics (MD) simulations were performed using the large-scale atomic/molecular massively parallel simulator (LAMMPS) code $^{35}$ with reactive force field (ReaxFF) potentials. ${ }^{32-34}$ The MD time step was set to $0.25 \mathrm{fs}$, which was determined from a stability test using a microcanonical ensemble (NVE). To properly account for the electron beam irradiation, heating was controlled during simulation by increasing the temperature from $1000 \mathrm{~K}$ to $4000 \mathrm{~K}$ for $1000 \mathrm{ps}$ using a canonical ensemble (NVT) and the Nose-Hoover chain thermostat.

\section{Acknowledgements}

This work was supported by the Nano Material Technology Development Program through the National Research Foundation of Korea (NRF) funded by the Ministry of Science, the ICT and Future Planning (2012M3A7B4049807, 2012M3A7B4049888) and Priority Research Center Program (2010-0020207) through NRF funded by the Ministry of Education (MOE), and the IBS-R019D1. We appreciate comments by P. Thrower.

\section{Notes and references}

1 F. Banhart, J. Kotakoski and A. V. Krasheninnikov, ACS Nano, 2010, 5, 26-41.

2 Ç. Ö. Girit, J. C. Meyer, R. Erni, M. D. Rossell, C. Kisielowski, L. Yang, C.-H. Park, M. Crommie, M. L. Cohen and S. G. Louie, Science, 2009, 323, 1705-1708.

3 T. Ishii, T. Sato, Y. Sekikawa and M. Iwata, J. Cryst. Growth, 1981, 52(Part 1), 285-289.

4 R. T. Paine and C. K. Narula, Chem. Rev., 1990, 90, 73-91.

5 N. Alem, R. Erni, C. Kisielowski, M. D. Rossell, W. Gannett and A. Zettl, Phys. Rev. B: Condens. Matter, 2009, 80, 155425.

6 C. Jin, F. Lin, K. Suenaga and S. Iijima, Phys. Rev. Lett., 2009, 102, 195505.

7 J. C. Meyer, A. Chuvilin, G. Algara-Siller, J. Biskupek and U. Kaiser, Nano Lett., 2009, 9, 2683-2689.

8 N. Alem, O. V. Yazyev, C. Kisielowski, P. Denes, U. Dahmen, P. Hartel, M. Haider, M. Bischoff, B. Jiang, S. G. Louie and A. Zettl, Phys. Rev. Lett., 2011, 106, 126102.

9 C. T. Pan, R. R. Nair, U. Bangert, Q. Ramasse, R. Jalil, R. Zan, C. R. Seabourne and A. J. Scott, Phys. Rev. B: Condens. Matter, 2012, 85, 045440.

10 N. Alem, Q. M. Ramasse, C. R. Seabourne, O. V. Yazyev, K. Erickson, M. C. Sarahan, C. Kisielowski, A. J. Scott, S. G. Louie and A. Zettl, Phys. Rev. Lett., 2012, 109, 205502.

11 L.-C. Yin, H.-M. Cheng and R. Saito, Phys. Rev. B: Condens. Matter, 2010, 81, 153407.

12 V. Derycke, P. Soukiassian, A. Mayne, G. Dujardin and J. Gautier, Phys. Rev. Lett., 1998, 81, 5868.

13 J. R. Heath, Q. Zhang, S. C. O’Brien, R. F. Curl, H. W. Kroto and R. E. Smalley, J. Am. Chem. Soc., 1987, 109, 359-363.

14 R. J. Lagow, J. J. Kampa, H.-C. Wei, S. L. Battle, J. W. Genge, D. A. Laude, C. J. Harper, R. Bau, R. C. Stevens, J. F. Haw and E. Munson, Science, 1995, 267, 362-367.

15 H. E. Troiani, M. Miki-Yoshida, G. A. Camacho-Bragado, M. A. L. Marques, A. Rubio, J. A. Ascencio and M. JoseYacaman, Nano Lett., 2003, 3, 751-755.

16 T. D. Yuzvinsky, W. Mickelson, S. Aloni, G. E. Begtrup, A. Kis and A. Zettl, Nano Lett., 2006, 6, 2718-2722.

17 X. Zhao, Y. Ando, Y. Liu, M. Jinno and T. Suzuki, Phys. Rev. Lett., 2003, 90, 187401.

18 O. Cretu, H.-P. Komsa, O. Lehtinen, G. Algara-Siller, U. Kaiser, K. Suenaga and A. V. Krasheninnikov, ACS Nano, 2014, 8, 11950. 
19 J. Kotakoski, C. H. Jin, O. Lehtinen, K. Suenaga and A. V. Krasheninnikov, Phys. Rev. B: Condens. Matter, 2010, 82, 113404.

20 G. Kim, A. R. Jang, H. Y. Jeong, Z. Lee, D. J. Kang and H. S. Shin, Nano Lett., 2013, 13, 1834-1839.

21 W. Zhou, M. Prange, M. Oxley, S. Pantelides, S. Pennycook, J. Nanda, C. Narula and J. Idrobo, Microsc. Microanal., 2011, 17, 1498-1499.

22 Q. M. Ramasse, R. Zan, U. Bangert, D. W. Boukhvalov, Y.-W. Son and K. S. Novoselov, ACS Nano, 2012, 6, 4063-4071.

23 A. Zobelli, C. P. Ewels, A. Gloter, G. Seifert, O. Stephan, S. Csillag and C. Colliex, Nano Lett., 2006, 6, 1955-1960.

24 A. Zobelli, A. Gloter, C. P. Ewels, G. Seifert and C. Colliex, Phys. Rev. B: Condens. Matter, 2007, 75, 245402.

25 G. Kresse and J. Furthmüller, Comput. Mater. Sci., 1996, 6, 15-50.

26 G. Kresse and J. Furthmüller, Phys. Rev. B: Condens. Matter, 1996, 54, 11169.
27 P. E. Blöchl, Phys. Rev. B: Condens. Matter, 1994, 50, 1795317979.

28 G. Kresse and D. Joubert, Phys. Rev. B: Condens. Matter, 1999, 59, 1758-1775.

29 S. Grimme, J. Comput. Chem., 2006, 27, 17871799.

30 W. Kohn and L. J. Sham, Phys. Rev., 1965, 140, A1133A1138.

31 J. P. Perdew, K. Burke and M. Ernzerhof, Phys. Rev. Lett., 1997, 78, 1396-1396.

32 S. Plimpton, J. Comput. Phys., 1995, 117, 1-19.

33 A. C. van Duin and J. S. S. Damsté, Org. Geochem., 2003, 34, 515-526.

34 A. C. T. van Duin, S. Dasgupta, F. Lorant and W. A. Goddard, J. Phys. Chem. A, 2001, 105, 93969409.

35 S. Cheng and G. S. Grest, LAMMPS Molecular Dynamics Simulator, http://lammps.sandia.gov/index.html. 